\title{
Anselme et Descartes, Deux Arguments Logico-théologiques
}

\author{
[Anselmo e Descartes, Dois Argumentos Lógico-teológicos]
}

Joseph Vidal-Rossett

Résumé: Ce texte est une version profondément remaniée d'une conférence donnée sur ce sujet en février 2018 lors de la journée d'études à Nancy, dans le cadre des Archives Vuillemin, et en mai 2018, à l'Université de Brasilia. Contrairement à ce que j'ai affirmée dans ces conférences ainsi que dans une publication précédente (Vidal-Rosset, 2013), la formalisation de l'argument de Descartes (que je développe ici en déduction naturelle) montre que la logique minimale ne suffit pas à traduire l'argument de Descartes; la logique intuitionniste est requise pour que cet argument soit valide. Si la traduction de l'argument d'Anselme que je donne pour commencer contredit l'analyse et les conclusions de Vuillemin sur ce sujet, en revanche, l'interprétation que Vuillemin fait du système de Descartes comme philosophie intuitionniste est confirmée par la formalisation qui suit. Enfin, le pluralisme philosophique propre à l'esprit de la classification de Vuillemin est aussi éclairé par la conclusion de cet article.

Mots clés: Déduction naturelle. Logique minimale. Logique intuitionniste. Pluralisme philosophique.

Resumo: Este texto é uma versão profundamente revisada de uma conferência sobre o mesmo assunto realizada em fevereiro de 2018, durante o dia de estudos em Nancy, no quadro dos Arquivos Vuillemin, e em maio de 2018, na Universidade de Brasília. Ao contrário do que afirmei nestas conferências e também em publicação anterior (VidalRosset, 2013), a formalização do argumento de Descartes (que desenvolvo aqui em uma dedução natural) mostra que a lógica mínima não é suficiente para traduzir o argumento de Descartes; a lógica intuicionista é necessária para que este argumento seja válido. Se a tradução do argumento de Anselmo, que apresento de início, contradiz a análise e as conclusões de Vuillemin sobre este assunto, por outro lado, a interpretação de Vuillemin do sistema de Descartes como uma filosofia intuicionista é confirmada pela formalização que segue. Finalmente, o pluralismo filosófico específico ao espírito da classificação de Vuillemin também é esclarecido pela conclusão deste artigo.

Palavras-chave: Dedução natural. Lógica mínima. Lógica intuicionista. Pluralismo filosófico.

\footnotetext{
${ }^{*}$ Membre titulaire du Département de philosophie de l'Université de Lorraine à Nancy. Maître de conférences HDR. Doctorat de Lettres et Sciences Humaines - Spécialité Philosophie et épistémologie, Université de Provence (Aix-Marseille I). E-mail: joseph.vidalrosset@univ-lorraine.fr. ORCID: https://orcid.org/0000-0001-6683-7629.
} 


\section{Introduction}

Cet article porte sur la structure logique des arguments a priori qu'Anselme et Descartes ont donnés pour prouver l'existence de Dieu. Rappelons deux définitions cruciales d'un point de vue logique:

Définition 1.1. - Un argument est déductivement valide si et seulement si sa conclusion est vraie chaque fois que toutes ses prémisses sont vraies. (Bonevac, 2003, p. 17)

Définition 1.2. - Un argument est correct si et seulement si

\section{1) il est valide}

2) toutes ses prémisses sont vraies. (Bonevac, 2003, p. 18)

La section 2 prouve que l'argument d'Anselme est valide en logique classique du premier ordre. La section 3 prouve que l'argument de Descartes est valide en logique intuitionniste du premier ordre, c'est-à-dire dans une logique plus faible que la logique classique. La question de la correction de ces deux arguments, et notamment celui de Descartes, n'est évidemment traitée qu'après la preuve de leur validité respective.

\section{L'argument d'Anselme}

La traduction formelle de l'argument d'Anselme a déjà souvent été donnée sous plusieurs formes (voir par exemple celle donnée par Tennant (Tennant, 2014, pp. 213-226), mais celle qui est donnée dans cet article est peut-être la plus simple qui soit, parce qu'elle est fondée sur la version la plus élégante de l'argument d'Anselme, celle donnée par Gaunilon (Anselme, 1993, p. 77):

Étant donné qu'on dit, à qui met en doute ou même nie qu'il y ait une certaine nature que rien ne se peut penser de plus grand, qu'il est prouvé qu'elle est de ce que, premièrement, celui-là même qui nie ou doute à son propos l'a déjà dans l'intellect puisque, l'entendant dite, il comprend ce qu'on dit; parce que, ensuite, ce qu'il comprend, il est nécessaire que ça ne soit pas seulement dans l'intellect, mais aussi dans la réalité, chose qu'on prouve de ce qu'il est plus grand d'être aussi dans la réalité que d'être seulement dans l'intellect, et que, si c'est seulement dans l'intellect, sera plus grand que tout ce qui aura été aussi dans la réalité, ainsi le plusgrand-que-tout sera moindre que quelque chose et ne sera pas le plus-grand-que-tout, ce qui assurément est contradictoire; et partant, il est nécessaire que le plus grand que tout, qu'on a déjà prouvé être dans l'intellect, ne soit pas seule- 
ment dans l'intellect mais soit aussi dans la réalité, puisque autrement il ne pourra pas être plus grand que tout.

Anselme a répondu à Gaunilon que l'expression "plus grand que tout doit être remplacée par "ce que rien de plus grand ne peut être pensé, mais cette correction n'affecte ni la validité ni l'élégance de la preuve d'Anselme, dans la version que Gaunilon en donne. Pour traduire celle-ci dans le langage de la logique du premier ordre [David; Nour; Raffalli, 2004, pp. 9-40], on se donne les règles de la déduction naturelle, dans le style adopté par von Plato (2013), ainsi que le lexique suivant:
- " $x>y$ " signifie " $x$ est [pensé comme étant] plus grand que $y$ ".

- “ $\neg(x>y)$ " signifie "il est faux que $x$ soit [pensé comme étant] plus grand que $y$ ".

- “ $d$ " est une constante d'individu qui remplace le nom propre Dieu.

- " $R d "$ signifie "Dieu est [pensé comme étant] quelque chose qui existe en réalité".

On peut remarquer que le prédicat "exister dans la pensée" est inutile, puisqu'il s'agit uniquement de prouver que le prédicat "exister en réalité" est vrai de Dieu. Pour en donner la preuve formelle, trois prémisses sont nécessaires, à savoir:

$$
\begin{gathered}
\forall y \neg(y>d) \\
\forall x \forall y((\neg R x \wedge R y) \rightarrow(y>x))
\end{gathered}
$$

$R a$

- La prémisse (1) est la définition de Dieu: l'être qui est tel que rien de plus grand ne peut être pensé.

- La prémisse (2) est la prémisse ajoutée par Gaunilon: tout ce qui est [pensé comme] une chose qui existe en réalité est aussi [pensé comme] plus grand que quoi que ce soit qui n'est pas pensé comme tel.
(Prémisse hérétique en conséquence de laquelle même la boue doit être pensée comme supérieure à Dieu, si Dieu, contrairement à la boue, n'existe pas en réalité.)

- La prémisse (3) dit que quelque chose existe en réalité.

L'argument d'Anselme se laisse alors traduire par le séquent suivant: 


$$
\forall y \neg(y>d), \forall x \forall y((\neg R x \wedge R y) \rightarrow(y>x)), R a \vdash R d
$$

Démonstration. -

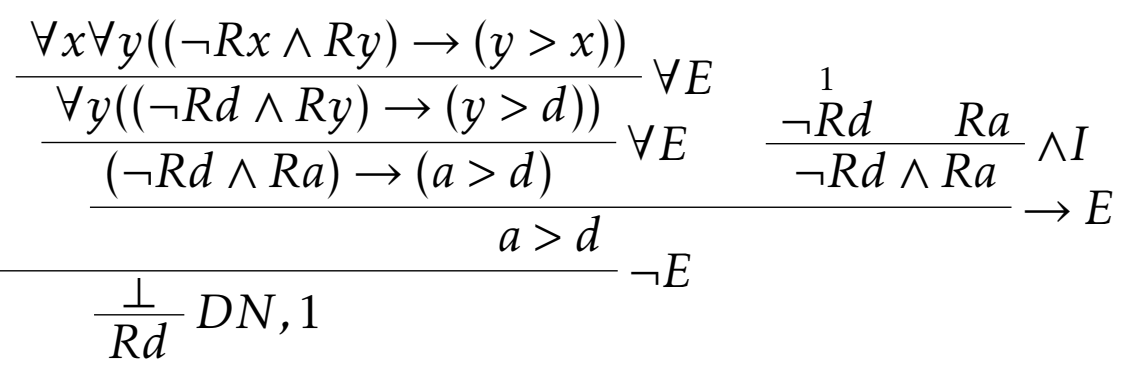

Cette formalisation de la preuve d'Anselme en logique du premier ordre permet trois remarques.

Remarque 2.1. - Contrairement à ce qui a été parfois écrit, l'argument d'Anselme n'implique aucun paradoxe. Il ne tombe ni sous le coup du paradoxe de Russell, comme l'a soutenu Viger (2002), ni sous celui de BuraliForti comme l'a cru Vuillemin (1971).

Remarque 2.2. - La décharge de la prémisse mineure (c'est-à-dire la décharge de $\neg R d$ ) qui est la négation de la thèse qui affirme que le prédicat d'existence est un prédicat de Dieu montre que l'argument d'Anselme est un argument ontologique ou, plus exactement, qu'il est une réduction à l'absurde de la négation de l'argument ontologique. Par conséquent, cet argument n'échappe ni à la critique destructrice de Kant qui soutient "l'impossibilité d'une preuve on- tologique de l'existence de Dieu (Kant, 2006, pp. 530-536), ni à la correction de bon sens que la logique libre de Lambert (Lambert, 2002, p. 123) apporte à la logique du premier ordre usuelle, en soulignant que ni un terme particulier (comme $d$ ) ni un terme général (comme $R$ ) ne peuvent par eux-mêmes et eux seuls avoir de conséquences existentielles.

Remarque 2.3. - Enfin, si le séquent (4) est valide, il ne l'est qu'en logique classique. En logique intuitionniste, l'usage de la règle d'introduction de la négation serait la dernière étape de la preuve ci-dessus, la règle spécifiquement classique de l'élimination de la double négation ne pourrait pas être utilisée, et donc la conséquence des prémisses mentionnées serait plus faible; autrement dit, le séquent (4) ne serait pas prouvable, mais le serait le séquent suivant:

$$
\forall y \neg(y>d), \forall x \forall y((\neg R x \wedge R y) \rightarrow(y>x)), R a \vdash \neg \neg R d
$$


Cette dernière remarque signifie que, d'un point de vue intuitionniste, si l'on oublie les objections décisives de Kant, la position de l'athée qui nie l'existence de Dieu serait effectivement réduite à l'absurde par l'argument d'Anselme, mais non celle de l'agnostique qui refuse de l'affirmer.

\section{L'argument de Descartes}

Voici le lexique utilisé plus bas pour formaliser la première preuve que Descartes donne de l'existence de Dieu dans la Méditation troisième:

- "c" signifie "le Cogito",

- " $d$ " signifie "l'idée de Dieu",

- “ $x \geqslant y$ " signifie "le degré de perfection de $x$ est au moins égal à celui de $y^{\prime \prime}$,
- "Sxy" signifie "la réalité formelle $x$ est la cause totale et efficient de la réalité objective $y^{\prime \prime}$ (la signification des expressions "réalité formelle" et "réalité objecive" est expliquée plus bas),

- "Rcy" signifie " $y$ est une pensée présente dans le Cogito",

- Enfin, au règles usuelles de la déduction naturelle pour la logique $\mathrm{du}$ premier ordre, j'ajoute les deux règles suivantes qui se déduisent toutes deux de la définition du symbole $\geqslant$, defm. et defs. étant les abréviations respectives de definiens et de de definiendum, car c'est le definiens du symbole $\geqslant$ qui est la conclusion de la règle à gauche et le symbole $\geqslant$, en tant que definien$d u m$, qui est la conclusion de la règle à droite de la table suivante:

$$
\frac{x \geqslant y}{(x>y) \vee(x=y)} \geqslant \text { defs. } \frac{(x>y) \vee(x=y)}{x \geqslant y} \geqslant \text { defm. }
$$

Le noyau de la preuve de Descartes est contenu dans cet alinéa (Descartes, 1641, pp. 115-117):

Partant il ne reste que la seule idée de Dieu, dans laquelle il faut considérer s'il y a quelque chose qui n'ait pu venir de moi-même. Par le nom de Dieu j'entends une substance infinie, éternelle, immuable, indépendante, toute connaissante, toute-puissante, et par laquelle moi-même, et toutes les autres choses qui sont (s'il est vrai qu'il y en ait qui ex- 
istent) ont été créées et produites. Or ces avantages sont si grands et si éminents, que plus attentivement je les considère, et moins je me persuade que l'idée que j'en ai puisse tirer son origine de moi seul. Et par conséquent, il faut nécessairement conclure de tout ce que j'ai dit auparavant que Dieu existe. Car, encore que l'idée de la substance soit en moi, de cela même que je suis une substance, je n'aurais pas néanmoins l'idée d'une substance infinie, moi qui suis un être fini, si elle n'avait été mise en moi par quelque substance qui fût véritablement infinie.

L'argument de Descartes est la déduction d'une conjonction de deux énoncés, déduction qui peut donc être divisée en deux preuves subalternes. Pour utiliser le vocabulaire de Descartes, la dernière phrase de la citation précédente signifie que la réalité objective de l'idée de substance peut être causée par la réalité formelle du Cogito parce que le Cogito est une substance, mais étant une substance finie, le Cogito ne peut pas être la cause de son idée de Dieu, car la réalité objective de cette idée est une substance infinie et seule une réalité formelle d'une substance infinie peut être la cause de cette représentation. Sur ce point, les explications de Williams (Williams, 2005, p. 125) sont lumineuses:

Descartes appelle "réalité formelle" la réalité que toute chose possède intrinsèquement, et "réalité objective" la réalité qu'une idée possède en vertu de son objet. Ainsi, toutes les idées ont le même degré de réalité formelle, mais elles ont des degrés de réalité objective différents, parce que leurs objets ont (ou auraient) différents degrés de réalité formelle. Enfin, en exprimant le principe selon lequel la cause de quelque chose doit contenir au moins autant de réalité que l'effet, il dit que la réalité de l'effet doit exister dans la cause soit formellement, soit éminemment: formellement, s'il y a autant de réalité dans la cause que dans l'effet; éminemment, s'il y a plus de réalité dans la cause que dans l'effet, la cause étant d'un type plus élevé que l'effet (ce sera le cas avec les œuvres d'art, l'esprit de l'artiste étant d'un type de réalité plus élevé que n'importe lequel de ses produits). En réunissant tous ces termes, le principe de Descartes sur la causalité des idées se présente ainsi: la cause de toute idée doit contenir soit formellement soit éminemment autant de réalité que l'idée possède formellement et objective- 
ment.

Dans sa plus simple expression, l'argument de Descartes a pour conclusion l'énoncé suivant: "le Cogito n'est pas la cause de l'idée de Dieu et il existe une chose qui est la cause de cette idée de Dieu et qui est aussi parfaite que celle-ci"

énoncé qui se traduit formellement par

$$
\neg S c d \wedge \exists x(S x d \wedge(x=d))
$$

Il y aura donc une première preuve subalterne pour déduire la formule qui est l'élément gauche de la conjonction (6), à savoir et une seconde preuve subalterne pour déduire la formule qui est le second élément de la conjonction (6).

\subsection{Première preuve subalterne. - Il} n'est besoin que de trois prémisses pour déduire (7), la première étant la définition de l'idée de Dieu, qui est l'idée d'une "substance infinie, éternelle, immuable, indépendante, toute connaissante, toute-puissante, et par laquelle moi-même, et toutes les autres choses qui sont (s'il est vrai qu'il y en ait qui existent) ont été créées et produites". La définition que Descartes donne de Dieu signifie bien que, pour le Cogito, il est impossible d'avoir l'idée d'un être supérieur à Dieu. En conséquence, la traduction formelle donnée plus haut de la définition anselmienne de Dieu doit pouvoir être utilisée dans le contexte cartésien, c'est donc la formule (1) qui sera la première prémisse de l'argument de Descartes, où $d$ est le nom de l'idée de Dieu, idée telle qu'aucune idée d'un être plus grand ne peut être pensée.

Contrairement à l'idée de Dieu, je peux toujours concevoir quelque chose de plus parfait que moi. Par conséquent, exprimé à la première personne, notre deuxième prémisse dirait "Je n'ai certainement pas toutes les perfections que je conçois dans l'idée de Dieu" et, dans notre lexique, cette deuxième prémisse dit "il est faux que le degré de perfection de le Cogito est le même que le degré de perfection de l'idée de Dieu: 


$$
\neg(c=d)
$$

Voici maintenant le développement ciliter la compréhension du lecteur. Red'une étape intermédiaire de la formal- marquons qu'à partir de (1) et de (8) il isation de l'argument qui devrait fa- est possible de déduite $\neg(c \geqslant d)$ :

$$
\forall y \neg(y>d), \neg(c=d) \vdash \neg(c \geqslant d)
$$

Démonstration. -

$$
\frac{(c>d) \vee(c=d) \frac{\frac{\forall y \neg(y>d)}{\neg(c>d)} \forall E \quad \stackrel{2}{\perp} d}{\perp} \neg E \frac{\neg(c=d) c \stackrel{2}{=} d}{\perp} \vee E, 2}{\frac{\perp((c>d) \vee(c=d))}{\neg(c \geqslant d)} \geqslant d, 1} \geqslant
$$

Il suffit ensuite de supprimer les hy- schéma précédent à cette déduction qui pothèses déchargées ainsi que les dé- va être une partie de la première preuve ductions intermédiaires pour réduire le subalterne:

$$
\frac{\frac{\forall y \neg(y>d)}{\neg(c>d)} \forall E \quad \neg(c=d)}{\neg(c \geqslant d)} D M i
$$

Concluons cette remarque en soulignant que ce séquent

$$
\neg(c>d), \neg(c=d) \vdash \neg((c>d) \vee(c=d))
$$

qui vient d'être prouvé est une instance l'étiquette choisie, DMi), alors qu'il est des lois de De Morgan qui est dériv- bien connu que cette autre instance des able en logique intuitionniste (d'où lois de De Morgan:

$$
\neg((c>d) \wedge(c=d)) \vdash \neg(c>d) \vee \neg(c=d)
$$


n'est pas valide en logique intuitionniste; or cette dernière déduction ne fait pas partie de l'argument de Descartes.

Enfin, une application spécifique du principe de causalité est faite par Descartes quand il écrit (Descartes, 1641, p. 107):

Maintenant, c'est une chose manifeste par la lumière naturelle, qu'il doit y avoir pour le moins autant de réalité dans la cause efficiente et totale que dans son effet: car d'où est-ce que l'effet peut tirer sa réalité sinon de sa cause ? et comment cette cause la lui pourrait-elle communiquer, si elle ne l'avait en elle-même. Et de là il suit, non seulement que le néant ne saurait produire aucune chose, mais aussi que ce qui est plus parfait, c'est-à-dire qui contient en soi plus de réalité, ne peut être une suite et une dépendance du moins parfait. Et cette vérité n'est pas seulement claire et évidente dans les effets qui ont cette réalité que les philosophes appellent actuelle ou formelle, mais aussi dans les idées où l'on considère seulement la réalité qu'ils nomment objective [...]

Appelons "principe de causalité appliquée" cette application du principe de causalité, des réalités formelles aux réalités objectives qui les représentent: "une réalité formelle $x$ est la cause d'une réalité objective $y$ seulement si le degré de perfection de $x$ est au moins égal à celui de $y^{\prime \prime}$, ce qui se traduit par:

$$
\forall x \forall y(S x y \rightarrow x \geqslant y)
$$

La formule (9) est la troisième et logique du premier ordre, la formule (7) dernière prémisse nécessaire pour construire la première preuve subalterne qui conclut que le Cogito n'est pas la cause de l'idée de Dieu. En effet, en est déductible de la conjonction de (1), (8) et (9), autrement dit le séquent suivant est prouvable:

$$
\forall y \neg(y>d), \neg(c=d), \forall x \forall y(S x y \rightarrow x \geqslant y) \vdash \neg S c d
$$

Démonstration. - 


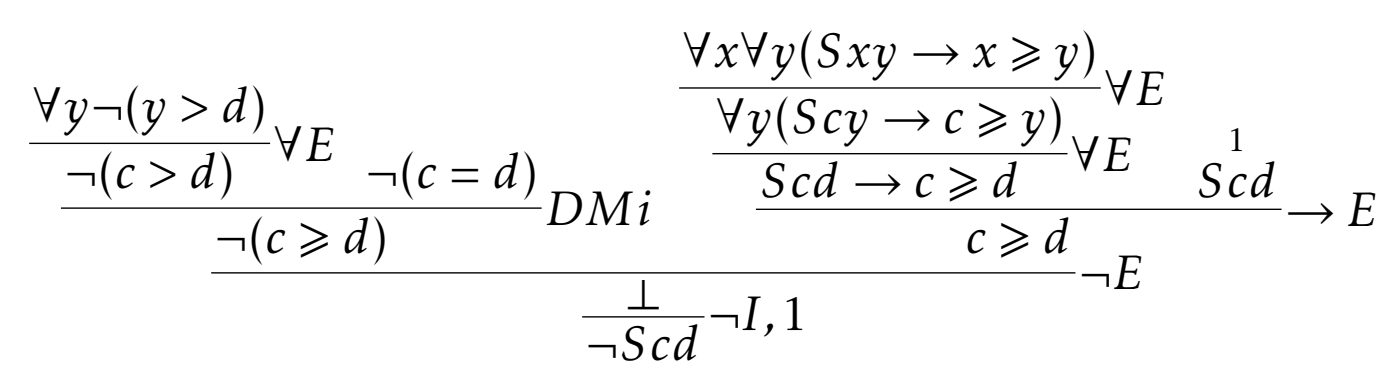

\subsection{Seconde preuve subalterne. -} Dans un deuxième temps, l'argument de Descartes doit prouver qu'il y a une réalité formelle qui est cause de l'idée de Dieu et que cette réalité formelle a nécessairement la même perfection que la réalité objective de cette idée. Puisqu'il vient d'être prouvé que le Cogito n'est pas la cause de l'idée de Dieu, il s'agit maintenant de prouver qu'il y a une cause à l'idée de Dieu - ce qui met fin au solipsisme - et que cette cause est Dieu, ce qui réfute le scepticisme. Dans le langage de la logique du premier ordre avec notre lexique, la seconde preuve subalterne de l'argument de Descartes doit être la déduction de la formule suivante qui se trouve à droite de la conjonction (6):

$$
\exists x(S x d \wedge(x=d))
$$

Déduire $\exists x(S x d)$ n'est pas difficile; "l'idée de Dieu est pensée par le Cogito", deux prémisses suffisent: "toute idée à énoncés qui se laissent traduire respeclaquelle le Cogito pense a une cause" et tivement par

$$
\forall y(R c y \rightarrow \exists x S x y)
$$

et par

$$
\text { Rcd }
$$

et à partir desquels on peut con- verselle et d'élimination du conditionclure $\exists x(S x d)$ en appliquant les règles nel:

d'élimination de la quantification uni-

$$
\frac{\forall y(R c y \rightarrow \exists x S x y)}{\frac{R c d \rightarrow \exists x S x d}{\exists x S x d} \quad R E \quad R c d} \rightarrow E
$$

À partir de ce dernier résultat, il est désormais possible de déduire (11) sans ajouter de prémisses, autrement dit, le séquent 


$$
\forall y(R c y \rightarrow \exists x S x y), R c d, \forall x \forall y(S x y \rightarrow x \geqslant y), \forall y \neg(y>d) \vdash \exists x(S x d \wedge(x=d))
$$

est prouvable (voir Appendice A, page 231).

Il devient alors trivial de déduire la conjonction (6) en appliquant la règle d'introduction de la conjonction à partir des preuves des séquents (10) et (14). Le séquent

$$
\forall y \neg(y>d), \neg(c=d), \forall x \forall y(S x y \rightarrow x \geqslant y), \forall y(R c y \rightarrow \exists x S x y), R c d \vdash \neg S c d \wedge \exists x(S x d \wedge(x=d))
$$

est donc évidemment prouvable à partir des deux preuves précédentes. Voici le début de la démonstration de (15): on laisse le lecteur avec la preuve du séquent 100 , page 225, pour la partie gauche de cette démonstration, et la preuve du séqent (14), page 231, pour la partie droite:

$$
\begin{aligned}
& \text { Démonstration. - }
\end{aligned}
$$

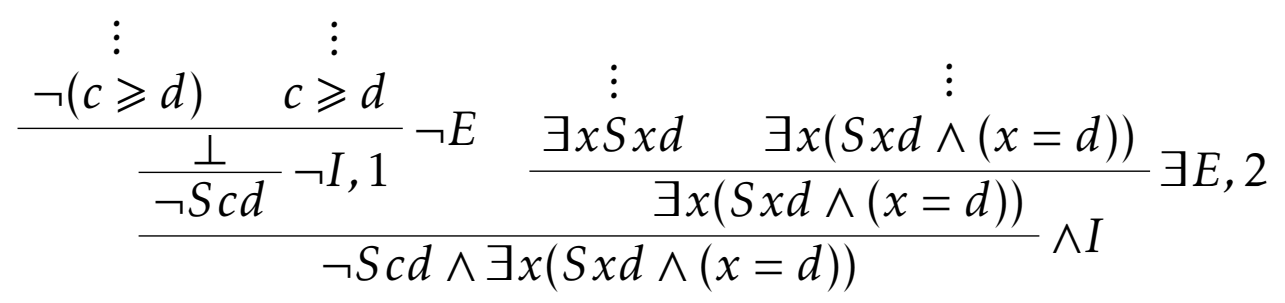

On laisse le lecteur compléter cette démonstration en se reportant à la preuve du séquent (10), page 225, pour la partie gauche, et la preuve du séquent (14), page 231, pour la partie droite.

\subsection{L'argument de Descartes est-il} correct? - Ce qui précéde démontre que la conclusion de l'argument de Descartes est incontestablement une conséquence logique des prémisses qu'il considère comme nécessaires ou vraies. L'argument de Descartes est déductivement valide et sa validité est démontrable en logique intuitionniste $\mathrm{du}$ premier ordre, par contraste avec l'argument d'Anselme dont la validité n'est prouvable qu'en logique classique, c'est-à-dire dans une logique plus forte que celle dont Descartes a besoin.

Si toutes les prémisses de l'argument de Descartes étaient vraies, alors son argument serait correct et il serait logiquement impossible de considérer sa conclusion comme pouvant être fausse. Cependant, en raison de la méthode du 
doute radical adoptée par Descartes, il faut rappeler ce qui est admis comme vrai par Descartes: est vraie toute idée qui ne peut sans contradiction être considérée comme métaphysiquement douteuse, il est par exemple contradictoire que je puisse douter que je pense au moment même où je doute, donc je pense. Par manque d'attention à l'ordre des raisons, on a pu croire à tort que Descartes est victime du cercle que, comme le remarque Doney (Doney, 1955, p. 325), Arnauld décrit avec concision dans les Quatrièmes Objections (Descartes, 1641, p. 337):

Il ne me reste plus qu'un scrupule, qui est de savoir comment il se peut défendre de ne pas commettre un cercle, lorsqu'il dit que nous ne sommes assurés que les choses que nous concevons clairement et distinctement sont vraies, qu'à cause que Dieu est ou existe.

Car nous ne pouvons être assurés que Dieu est, sinon parce que nous concevons cela très clairement et très distinctement; donc, auparavant que d'être assurés de l'existence de Dieu, nous devons être assurés que toutes les choses que nous concevons clairement et distinctement sont toutes vraies.
Mais Descartes répond à Arnauld qu'il est faux qu'il accepte la thèse de la vérité de toutes les idées claires et distinctes avant que l'existence de Dieu ne soit prouvée (Descartes, 1641, p. 369). En effet, la preuve de l'existence de Dieu ne se fonde que sur les raisons de la lumière naturelle, c'est-à-dire, comme le souligne Rose (Rose, 1965, p. 80), sur les idées métaphysiquement certaines qui, par définition et à l'instar du "Je pense", sont invincibles à tout le pyrrhonisme dépendant de l'hypothèse du mauvais génie. La méthode de Descartes consiste donc à s'appuyer uniquement sur des idées métaphysiquement certaines, c'est-à-dire sur des idées dont on ne peut sans absurdité admettre la possible fausseté, pour prouver l'existence d'au moins une chose extérieure au Cogito, à savoir Dieu. Par conséquent, l'argument de Descartes est incorrect seulement si au moins une des deux conditions suivantes est satisfaite:

(a) il est prouvable que l'une des prémisses $p$ de l'argument est métaphysiquement douteuse, c'està-dire que $p$ peut-être rejetée parce que $p$ n'échappe pas à l'hypothèse du mauvais génie telle que Descartes l'a formulée,

(b) il est prouvable que $p$ ne respecte pas le principe de la suspension du jugement, parce que $p$ porte jugement sur la réalité formelle d'une chose à partir de la représentation de celle-ci, avant que la démonstra- 
tion de l'existence de Dieu (garant de la vérité des idées claires et distinctes) ne soit faite.

Si l'analyse développée plus haut est fidèle, il n'y a que cinq prémisses à examiner pour décider de la correction de l'argument de Descartes. Commençons par juger des trois prémisses qui, une fois formalisées, contiennent un quantificateur universel, à savoir (1), (9) et (12), c'est-à-dire la définition de l'idée de Dieu, et les deux prémisses qui mentionnent le principe de causalité.

Hobbes conteste l'existence d'une idée de Dieu comparable à celle que Descartes formule (Descartes, 1641, pp. 304-307). Mais si l'on affirme que la réalité objective de l'idée de Dieu n'est rien d'autre que cette signification que l'on accorde au mot "Dieu", alors on peut admettre le rejet de l'objection de Hobbes, ce qui revient à accorder que la prémisse (1) n'est ni métaphysiquement douteuse, ni ne contredit la suspension du jugement. On peut en effet admettre qu'il serait contradictoire d'entendre par Dieu un être tel que quelque chose de plus grand pourrait être pensé (puisque l'on a admis que la définition de Dieu donnée par Anselme s'accorde avec celle dont Descartes fait usage).

Il n'est pas non plus possible de rejeter les prémisses (9) et (12) sans nier ce qui les justifie, à savoir l'évidence "le néant ne saurait produit aucune chose". Supposons en effet qu'une représenta- tion possède plus de perfections que sa cause efficiente et totale, alors il faudrait que ces perfections ait pour cause le néant, ce qui est absurde. Il est donc nécessaire d'admettre les prémisses (9) et (12).

Restent les prémisses (8) et (13) qui ont la forme d'énoncés factuels. Il est déraisonnable de rejeter la prémisse (13) qui affirme modestement que le Cogito a une représentation de Dieu. Rejeter cette prémisse reviendrait aussi à rejeter la définition même de Dieu, c'est-à-dire (1).

Qu'en est-il enfin de la prémisse (8)? Mal interprétée, la prémisse (8) semble évidente si l'on se borne à lui faire dire "le Cogito n'est pas Dieu", mais ce n'est pas ce qu'elle dit. Rappelons sa signification précise: elle affirme que le degré de perfection du Cogito est nécessairement différent de celui de l'idée de Dieu, ce qui se justifie ainsi: je doute, donc ma perfection ne peut être comparable à celle qui est perçue dans l'idée de Dieu: la chose pensante que je suis ne peut pas être d'une perfection actuellement infinie. À ce sujet, Gueroult écrit (Gueroult, 1953, vol 1, p. 189):
Toute l'argumentation s'effon- drerait si l'on pouvait démon- trer que je suis infini et, par con- séquent, capable de produire par moi-même l'idée d'infini.

Mais c'est soumettre la réfutation de l'argument à une condition qui est à 
l'évidence trop forte, car celle-ci ne se fonde pas sur la méthode du doute. La condition étant trop forte, elle pourrait laisser dans l'ombre une faille de l'argument. On corrige donc la condition énoncée par Gueroult la façon suivante: l'argument de Descartes s'effondre s'il est impossible d'écarter l'hypothèse qu'une puissance infinie me trompe et fait que j'imagine être fini, alors que je suis actuellement infini. Autrement dit, il suffit qu'il soit possible de prouver que la prémisse (8) est métaphysiquement douteuse pour que l'argument de Descartes puisse être jugé irrecevable, en raison même de la méthode du doute. Or si la puissance du mauvais génie est telle qu'il est capable de faire en sorte que je me trompe en accordant une valeur objective aux opérations arithmétiques les plus simples, pourquoi serait-elle incapable de me tromper lorsque je pense être fini?

On imagine pouvoir répondre à une telle objection en rappelant que l'on ne conçoit pas Dieu comme pouvant être victime de tromperie, car sinon, on contredirait la prémisse (1), puisque Dieu ne serait plus ce maximum de perfection exprimé par celle-ci. C'est ainsi que l'on peut croire que la première preuve subalterne est saine et sauve.

Malheureusement, une telle réponse est à son tour réfutable, car d'une part, la prémisse (1) n'affirme pas qu'aucun être n'est égal à Dieu; d'autre part, il est conforme à la méthode du doute métaphysique d'accorder au mauvais génie une puissance comparable à celle que l'on suppose en Dieu, car il n'y a $a$ priori aucune raison d'accorder moins de puissance au Mal qu'au Bien. Dès lors, si j'accorde au mauvais génie une puissance infiniment trompeuse, alors, même si je suppose avoir toutes les qualités qui définissent Dieu, je ne peux décider si le mauvais génie échoue ou s'il réussit à me tromper. Par conséquent, je dois déclarer qu'en raison de la fiction méthodologique du mauvais génie, j'ignore si ma perfection est égale ou inférieure à celle de Dieu. La condition (a) étant satisfaite, il est démontré que l'argument de Descartes est incorrect.

Enfin, si la preuve formelle développée plus haut est une traduction fidèle de l'argument de Descartes, alors la condition (b) est également satisfaite, ce qui décide également du caractère incorrect de l'argument de Descartes. En effet, pour que la première preuve subalterne soit concluante, le principe de causalité exprimé par la prémisse (9) doit non seulement définir un rapport entre réalité formelle et réalité objective, mais il faut aussi que la prémisse (8) porte sur la réalité formelle du Cogito et donc trahisse la méthode de suspension du jugement. On voit donc ici que la fameuse objection de Kant qui dénonce un paralogisme dans le passage du Cogito à l'affirmation de l'existence de la res cogitans (Kant, 2006, pp. 360-416) est parfaitement justifiée et ici formellement prouvée. 
4. Conclusion: intuitionnisme et scepticisme

Si la formalisation de l'argument d'Anselme fragilise l'analyse que Vuillemin (1971) en a faite, celle de l'argument de Descartes confirme et donc renforce l'interprétation qu'il donne de la philosophie de Descartes comme exemple de système philosophique intuitionniste. Mais il y a plus. L'échec de "la première preuve par les effets" (appellation que Gueroult donne à cet argument de Descartes) apparaît comme une justification du pluralisme philosophique de Vuillemin, car cet échec suggère qu'aucune preuve intuitionniste ne peut réfuter la position sceptique en contraignant le sceptique à renoncer à la suspension du jugement. Contrairement au sceptique, l'intuitionniste postule que certaines apparences ou représentations sont des vérités au sujet d'une réalité qui n'est pas mentale. En raison de ce postulat qui s'ajoute en quelque sorte au privilège que l'intuitionniste accorde aux jugements de méthode (Vuillemin, 1981; 1984; 1986), "l'intuitionniste reste un dogmatique aux yeux du sceptique", pour reprendre un mot de Vuillemin, lors d'un cours au Collège de France.

\section{Appendice A}

$$
\forall y(R c y \rightarrow \exists x S x y), R c d, \forall x \forall y(S x y \rightarrow x \geqslant y), \forall y \neg(y>d) \vdash \exists x(S x d \wedge(x=d))
$$

Démonstration. -

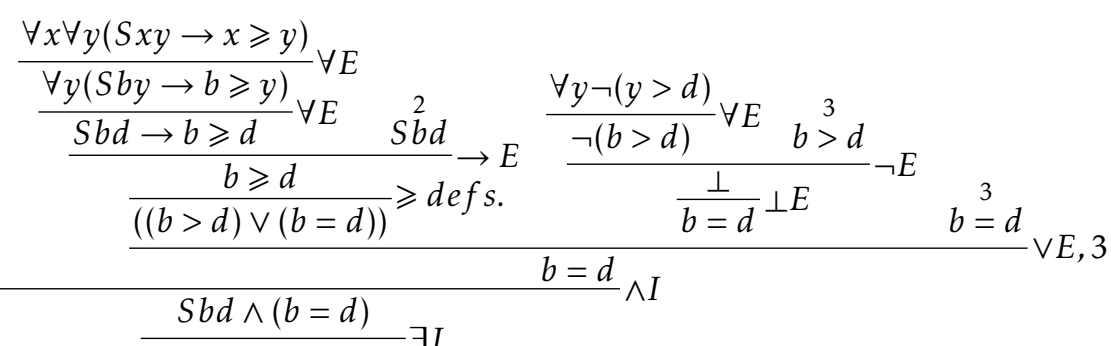

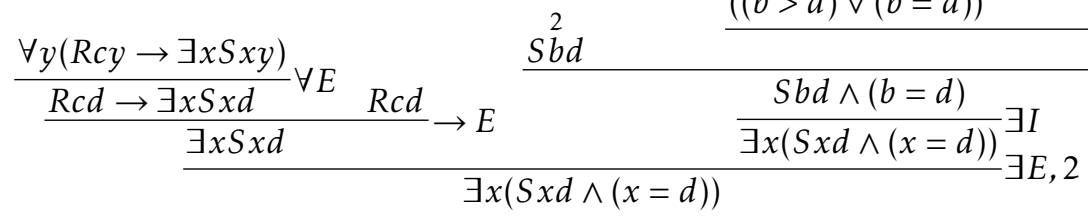




\section{Références}

ANSELME (1993). Proslogion: Allocution sur l'existence de Dieu. Flammarion, Paris, France. Traduction, préface et notes de Bernard Pautrat.

BONEVAC, D. (2003). Deduction: Introductory Symbolic Logic. Blackwell Pub., Malden, U.S.A.

DAVID, R., NOUR, K., and RAFFALLI, C. (2004). Introduction à la logique: théorie de la démonstration. Dunod, Paris.

DESCARTES, R. (1641). Méditations métaphysiques, Objections et Réponses. GF Flammarion, Paris, 2011. Présentation, bibliographie et chronologie par Jean-Marie Beyssade et Michelle Beyssade.

DONEY, W. (1955). The Cartesian Circle. Journal of the History of Ideas, 16(3): 324-338.

GUEROULT, M. (1953). Descartes selon l'ordre des raisons, volume I, II. Aubier, Paris, France.

KANT, E. (2006). Critique de la raison pure. Flammarion, impr. 2006, Paris, France.

LAMBERT, K. (2002). Free Logic: Selected Essays. Cambridge University Press, Cambridge, UK; New York.

ROSE, L. E. (1965). The Cartesian Circle. Philosophy and Phenomenological Research, 26(1): 80-89.

TENNANT, N. (2014). Introducing Philosophy. Routledge, New York, London.

VIDAL-ROSSET, J. (2013). Preuves intuitionnistes touchant la première philosophie. In LEPAGE, F., editor, La crise des fondements: quelle crise?, pages 149-184, Montréal, Canada. Département de philosophie de l'Université de Montréal, Les Cahiers d'Ithaque.

VIGER, C. (2002). St. Anselm's Ontological Argument Succumbs to Russell's Paradox. International Journal for Philosophy of Religion, 52(3): 123-128.

VON PLATO, J. (2013). Elements of Logical Reasoning. Cambridge University Press.

VUILLEMIN, J. (1971). Le Dieu d'Anselme et les apparences de la raison, volume 14 of Collection Analyse et Raisons. Aubier Montaigne, Paris.

VUILlEMIN, J. (1981). Trois philosophes intuitionnistes: Epicure, Descartes et Kant. Dialectica, 35: $21-41$.

VUILlEMIN, J. (1984). Nécessité ou contingence: l'aporie de Diodore et les systèmes philosophiques. Ed. de Minuit, Paris.

VUILlEMIN, J. (1986). What Are Philosophical Systems? Cambridge University Press, first edition edition.

Reçu / Recebido: 18/04/2020

Approuvé / Aprovado: 20/04/2020

Publié / Publicado: 20/09/2020 